\title{
Dominant Strategy Implementation in Economic Environments*
}

\author{
HideKi MiZUKAMI ${ }^{\dagger}$ \\ Faculty of Economics, University of Toyama \\ mizukami@eco.u-toyama.ac.jp \\ and \\ TAKUMA WAKAYAMA \\ Faculty of Urban Liberal Arts, Tokyo Metropolitan University \\ t_waka@center.tmu.ac.jp
}

November 21, 2006

\footnotetext{
*An earlier version of this paper was entitled "Dominant Strategy Implementation in Pure Exchange Economies." We are especially grateful to Tomas Sjöström, whose note led to improvements of the contents. We are also grateful to Masaki Aoyagi, Atsushi Kajii, Tatsuyoshi Saijo, Toyotaka Sakai, James Schummer, Koji Takamiya, Yoshikatsu Tatamitani, Naoki Yoshihara, an associate editor, and two anonymous referees for their invaluable comments and suggestions. Finally, we thank seminar participants at Kyoto University, the 10th Decentralization Conference in Japan, the 2004 Spring Meeting of the Japanese Economic Association, the 7th International Meeting of the Society for Social Choice and Welfare, Osaka Prefecture University, Osaka University, and Hitotsubashi University for their comments.

${ }^{\dagger}$ The author gratefully acknowledges financial support from the Ministry of Education, Culture, Sports, Science, and Technology, Grant-in-Aid for 21st Century COE Program "Interfaces for Advanced Economic Analysis."

${ }^{\ddagger}$ Corresponding author. Faculty of Economics, University of Toyama, 3190 Gofuku, Toyama 930-8555, JAPAN.
} 


\begin{abstract}
We study dominant strategy implementation especially in economic environments. We first show that in general environments, strategy-proofness and quasi-strong-non-bossiness together are necessary and sufficient for dominant strategy implementation via the associated direct revelation mechanism. We next prove that in weak separable environments, strategy-proofness is sufficient for dominant strategy implementation, by using an augmented revelation mechanism similar to the one devised by Jackson et al. (1994). Moreover, we focus on pure exchange economies without free disposal, and try to construct another augmented revelation mechanism that satisfies balancedness in and out of equilibrium, and which implements all strategy-proof social choice functions in dominant strategy equilibria.
\end{abstract}

Keywords: Augmented Revelation Mechanism, Strategy-Proofness, Balancedness, Quasi-Strong-Non-Bossiness, The Revelation Principle.

JEL Classification Numbers: D51, C72, D71, D78. 


\section{Introduction}

The revelation principle asserts that if a social choice function is implementable in dominant strategy equilibria, then it is truthfully implementable in dominant strategy equilibria, i.e., truthful revelation by each agent is a dominant strategy equilibrium of the associated direct revelation mechanism. However, the mechanism might have an untruthful dominant strategy equilibrium, too. So, the social choice function might not be fully dominant strategy implemented by the associated direct revelation mechanism (e.g., see Dasgupta et al. (1979) for dominant strategy implementation, and Postlewaite and Schmeidler (1986), Repullo (1986), and Palfrey and Srivastava (1989) for Bayesian implementation). This is called the multiple equilibrium problem. ${ }^{1}$

Another important topic on the revelation principle is to explore the relationship between implementation in dominant strategy equilibria and truthful implementation in dominant strategy equilibria. The revelation principle states that, in order for a social choice function to be dominant strategy implemented, it is necessary for it to be truthfully implemented in dominant strategy equilibria. In general, however, it is still an open problem whether truthful implementability (i.e., strategy-proofness) is a sufficient condition for dominant strategy implementation.

These problems do not arise when indifference in preferences is not allowed, because three notions of dominant strategy implementation-truthful implementation, implementation by the associated direct revelation mechanism, and implementation with indirect mechanisms-are equivalent in terms of implementability. In this case, the revelation principle has a powerful implication: in order to find out dominant strategy implementable social choice functions, it is necessary and sufficient to search for social choice functions that are truthfully implementable in dominant strategy equilibria. ${ }^{2}$ However, when indifference is possible, the revelation principle might lose the powerful implication. This is because the equivalence might not hold in general: there might be a truthfully implementable social choice function that cannot be fully dominant strategy implemented not only by the associated direct revelation mechanism but also by any indirect mechanism. In this paper, we explore the relationships among three notions of dominant strategy implementation to examine the implication of the revelation principle in economic environments.

This paper closely relates to two papers: one by Jackson et al. (1994) and

\footnotetext{
${ }^{1}$ The multiple equilibrium problem in dominant strategy implementation might not be disturbing, because, to quote Dasgupta et al. (1979), "[i]n direct mechanisms where telling the truth is one of several dominant strategies, it may be reasonable to suppose that players will in fact tell the truth." (See also Chapter 23 in Mas-Colell et al. (1995).) However, since they are each indifferent among dominant strategies irrespective of messages of the others, there is no guarantee that they will play the truthful dominant strategy. Indeed, recent experimental results indicate that they can choose a spiteful strategy (e.g., see Saijo (2005) for details).

${ }^{2}$ This is because every social choice function that is truthfully implementable in dominant strategy equilibria is fully implementable in dominant strategy equilibria.
} 
the other by Sjöström (1994). They showed that in separable environments, almost all social choice functions can be undominated Nash implemented by their bounded mechanisms. This might make us think that in separable environments, all of the strategy-proof social choice functions can be dominant strategy implemented by their mechanisms. Indeed, almost all strategy-proof social choice functions are implementable in dominant strategy equilibria by their mechanisms. However, not all strategy-proof social choice functions can be dominant strategy implemented by their mechanisms in separable environments: ${ }^{3}$ their mechanisms cannot dominant strategy implement any social choice function that assigns a common worst alternative for a preference profile. In contrast, we construct an augmented revelation mechanism similar to the one devised by Jackson et al. (1994), and show in Theorem 3 that our mechanism can dominant strategy implement every strategy-proof social choice function in weak separable environments. This is a partial answer to the open problem mentioned above.

Weak separable environments include pure exchange economies as special cases. So, Theorem 3 implies that in pure exchange economies, all of the strategyproof social choice functions can be dominant strategy implemented by our mechanism if strict value distinction is satisfied. Our mechanism as well as the one devised by Jackson et al. (1994) requires free disposability to ensure feasibility out of equilibrium. In pure exchange economies, however, a "natural" mechanism should be balanced in and out of equilibrium, as pointed out by Saijo et al. (1996). So, we next focus on pure exchange economies, and try to devise another augmented revelation mechanism satisfying balancedness in and out of equilibrium. In Theorem 5, we show that in pure exchange economies without free disposal, the new mechanism can implement every strategy-proof social choice function in dominant strategy equilibria, provided that there are three or more agents and that strict value distinction is satisfied.

This paper is also related to one by Jackson (1992). Jackson (1992) considered implementation in undominated strategies by a bounded mechanism, and showed that strategy-proofness is a necessary condition for bounded implementation in undominated strategies. However, Jackson (1992) left open the question of what is a sufficient condition for implementation in undominated strategies by a bounded mechanism. We provide a partial answer to this question by showing that in economic environments, strategy-proofness is both necessary and sufficient for bounded implementation via undominated strategies (See Theorems 4 and 6).

The robustness of implementation has recently deserved attention in implementation theory (e.g., see Bergemann and Morris (2005) for details). From this point of view, dominant strategy implementation has an advantage. This is because dominant strategy implementation does not need strong assumptions on

\footnotetext{
${ }^{3}$ This is why it has not yet been proved that strategy-proofness is a sufficient condition for dominant strategy implementation in separable environments.
} 
the information structure: agents need neither common knowledge nor common prior beliefs about each other's preferences.

This paper is organized as follows. Section 2 gives notation and definitions. In Section 3, we characterize the class of social choice functions, each of which can be dominant strategy implemented by the associated direct revelation mechanism in general environments. In Section 4, we demonstrate that the use of an indirect mechanism expands the class of dominant strategy implementable social choice functions. In Sections 5 and 6, we examine a sufficient condition for dominant strategy implementation with indirect mechanisms in weak separable environments and in pure exchange economies, respectively. Section 7 contains some concluding remarks. In the appendix, we briefly study a condition that is sufficient for dominant strategy implementation in general environments with a common worst alternative.

\section{Notation and Definitions}

Let $N:=\{1,2, \ldots, n\}$ be the set of agents, where $2 \leq n<+\infty$. The set of alternatives is denoted by $A$.

Each agent $i \in N$ has preferences over $A$, which are represented by a complete and transitive binary relation $R_{i}$. The strict preference relation and indifference relation associated with $R_{i}$ are denoted by $P_{i}$ and $I_{i}$, respectively. Let $\mathscr{R}_{i}$ denote the set of possible preferences for agent $i \in N$. A domain is denoted by $\mathscr{R}:=$ $\mathscr{R}_{1} \times \mathscr{R}_{2} \times \cdots \times \mathscr{R}_{n}$. A preference profile is a list $R=\left(R_{1}, R_{2}, \ldots, R_{n}\right) \in \mathscr{R}$.

An environment is a collection $(N, A, \mathscr{R})$.

Let $L C_{i}\left(a ; R_{i}\right):=\left\{b \in A \mid a R_{i} b\right\}$ be agent $i$ 's lower contour set of $a \in A$ at $R_{i} \in$ $\mathscr{R}_{i}$. For each agent $i \in N$, let $\arg \max _{\bar{A}} R_{i}:=\left\{a \in \bar{A} \mid a R_{i} b\right.$ for all $\left.b \in \bar{A}\right\}$ be the set of maximal elements in $\bar{A} \subseteq A$ at $R_{i} \in \mathscr{R}_{i}$. Note that $\arg \max _{\bar{A}} R_{i} \neq \varnothing$ for every $i \in N$ and every $R_{i} \in \mathscr{R}_{i}$ if $\bar{A}$ is finite, because $R_{i}$ is complete and transitive.

A social choice function is a single-valued function $f: \mathscr{R} \rightarrow A$ that assigns an alternative $a \in A$ to each preference profile $R \in \mathscr{R}$.

Let $M_{i}$ denote a message or strategy space of agent $i \in N$. We call $m_{i} \in M_{i}$ a message or strategy of agent $i \in N$. A mechanism is a pair $\Gamma=(M, g)$, where $M:=$ $M_{1} \times M_{2} \times \cdots \times M_{n}$ and $g: M \rightarrow A$ is an outcome function. Given a social choice function $f$, a mechanism $(M, g)$ is the associated direct revelation mechanism if $M_{i}=\mathscr{R}_{i}$ for every $i \in N$ and $g=f$. A message or strategy profile is denoted by $m=\left(m_{1}, m_{2}, \ldots, m_{n}\right) \in M$.

A strategy $m_{i}^{*} \in M_{i}$ is a dominant strategy of mechanism $(M, g)$ at $R_{i} \in \mathscr{R}_{i}$ if $g\left(m_{i}^{*}, m_{-i}\right) R_{i} g\left(m_{i}^{\prime}, m_{-i}\right)$ for all $m_{i}^{\prime} \in M_{i}$ and all $m_{-i} \in M_{-i}$. Let $D S_{i}^{\Gamma}\left(R_{i}\right) \subseteq M_{i}$ be the set of agent $i$ 's dominant strategies of mechanism $\Gamma=(M, g)$ at $R_{i} \in \mathscr{R}_{i}$.

A strategy profile $m^{*}=\left(m_{1}^{*}, m_{2}^{*}, \ldots, m_{n}^{*}\right) \in M$ is a dominant strategy equilibrium of mechanism $(M, g)$ at $R \in \mathscr{R}$ if, for any $i \in N, g\left(m_{i}^{*}, m_{-i}\right) R_{i} g\left(m_{i}^{\prime}, m_{-i}\right)$ for any $m_{i}^{\prime} \in M_{i}$ and any $m_{-i} \in M_{-i}$. Let $D_{S E}^{\Gamma}(R) \subseteq M$ be the set of all dominant strategy equilibria of mechanism $\Gamma=(M, g)$ at $R \in \mathscr{R}$. Let $g\left(D S E^{\Gamma}(R)\right):=$ 
$\left\{a \in A \mid a=g(m)\right.$ for some $\left.m \in D S E^{\Gamma}(R)\right\}$ be the set of dominant strategy equilibrium outcomes of mechanism $\Gamma=(M, g)$ at $R \in \mathscr{R}$. With an abuse of notation, we sometimes write $g\left(D S E^{\Gamma}(R)\right)=g\left(D S_{i}^{\Gamma}\left(R_{i}\right), D S_{-i}^{\Gamma}\left(R_{-i}\right)\right)$.

A mechanism $\Gamma=(M, g)$ dominant strategy implements a social choice function $f$ if $g\left(D S E^{\Gamma}(R)\right)=f(R)$ for any $R \in \mathscr{R} .^{4}$ A social choice function $f$ is implementable in dominant strategy equilibria if there exists a mechanism $\Gamma=(M, g)$ such that $g\left(D S E^{\Gamma}(R)\right)=f(R)$ for all $R \in \mathscr{R}$.

Now we introduce two properties of social choice functions. Strategy-proofness is an incentive compatibility requirement that no agent should be able to change her preferences in a way that results in a direct gain to her, irrespective of the other agents' preferences.

Definition 1 (Strategy-Proofness). A social choice function $f$ satisfies strategyproofness if, for all $R \in \mathscr{R}$ and all $i \in N$, there is no $R_{i}^{\prime} \in \mathscr{R}_{i}$ such that

$$
f\left(R_{i}^{\prime}, R_{-i}\right) P_{i} f(R) .
$$

Quasi-strong-non-bossiness, ${ }^{5}$ which is a version of non-bossiness, ${ }^{6}$ requires that if a change in one agent's preferences keeps her utility unchanged irrespective of the other agents' preferences, then it should not change the alternative.

Definition 2 (Quasi-Strong-Non-Bossiness). A social choice function $f$ satisfies quasi-strong-non-bossiness if, for all $R \in \mathscr{R}$, all $i \in N$, and all $R_{i}^{\prime} \in \mathscr{R}_{i}$, if $f\left(R_{i}, R_{-i}^{\prime \prime}\right) I_{i} f\left(R_{i}^{\prime}, R_{-i}^{\prime \prime}\right)$ for all $R_{-i}^{\prime \prime} \in \mathscr{R}_{-i}$, then

$$
f(R)=f\left(R_{i}^{\prime}, R_{-i}\right) .
$$

Remark 1. Quasi-strong-non-bossiness is weaker than strong-non-bossiness, ${ }^{7}$ which is a stronger version of non-bossiness.

\section{Implementation by the Direct Mechanism}

In this section, we identify necessary and sufficient conditions for a social choice function to be dominant strategy implemented by the associated direct revelation mechanism in general environments.

We begin by providing a necessary condition for dominant strategy implementation via the associated direct revelation mechanism.

\footnotetext{
${ }^{4}$ To simplify notation, we write $g\left(D S E^{\Gamma}(R)\right)=\{f(R)\}$ as $g\left(D S E^{\Gamma}(R)\right)=f(R)$.

${ }^{5}$ Quasi-strong-non-bossiness was called weak non-bossiness by Saijo et al. (2005).

${ }^{6}$ A social choice function $f$ satisfies non-bossiness if, for all $R \in \mathscr{R}$, all $i \in N$, and all $R_{i}^{\prime} \in \mathscr{R}_{i}$, if $f_{i}(R)=f_{i}\left(R_{i}^{\prime}, R_{-i}\right)$, then $f(R)=f\left(R_{i}^{\prime}, R_{-i}\right)$.

${ }^{7}$ Ritz (1983) first introduced the notion of strong-non-bossiness, called non-corruptibility. A social choice function $f$ satisfies strong-non-bossiness if, for all $R \in \mathscr{R}$, all $i \in N$, and all $R_{i}^{\prime} \in \mathscr{R}_{i}$, if $f(R) I_{i} f\left(R_{i}^{\prime}, R_{-i}\right)$, then $f(R)=f\left(R_{i}^{\prime}, R_{-i}\right)$.
} 
Theorem 1. If a social choice function $f$ is dominant strategy implementable by the associated direct revelation mechanism in general environments, then it satisfies quasi-strong-non-bossiness.

Proof. Let $\Gamma:=(\mathscr{R}, f)$. For each $R \in \mathscr{R}$ and each $i \in N$, let $f\left(D S_{i}^{\Gamma}\left(R_{i}\right), R_{-i}\right):=$ $\left\{a \in A \mid a=f\left(R_{i}^{\prime}, R_{-i}\right)\right.$ for some $\left.R_{i}^{\prime} \in D S_{i}^{\Gamma}\left(R_{i}\right)\right\}$.

Pick any $R \in \mathscr{R}$, any $i \in N$, and any $R_{i}^{\prime} \in \mathscr{R}_{i}$ such that

$$
f\left(R_{i}, R_{-i}^{\prime \prime}\right) I_{i} f\left(R_{i}^{\prime}, R_{-i}^{\prime \prime}\right) \text { for any } R_{-i}^{\prime \prime} \in \mathscr{R}_{-i} .
$$

Since $f$ is dominant strategy implementable by $\Gamma$, it holds that

$$
f\left(D S E^{\Gamma}\left(R^{\prime \prime}\right)\right)=f\left(R^{\prime \prime}\right) \text { for all } R^{\prime \prime} \in \mathscr{R} .
$$

Notice that (2) implies that $f\left(D_{S} E^{\Gamma}\left(R^{\prime \prime}\right)\right)$ is a singleton for any $R^{\prime \prime} \in \mathscr{R}$.

Substituting (2) for $f$ in (1) yields $f\left(D S_{i}^{\Gamma}\left(R_{i}\right), D S_{-i}^{\Gamma}\left(R_{-i}^{\prime \prime}\right)\right) I_{i} f\left(D S_{i}^{\Gamma}\left(R_{i}^{\prime}\right), D S_{-i}^{\Gamma}\left(R_{-i}^{\prime \prime}\right)\right)$ for all $R_{-i}^{\prime \prime} \in \mathscr{R}_{-i}$. Since $f\left(D S_{i}^{\Gamma}\left(R_{i}\right), D S_{-i}^{\Gamma}\left(R_{-i}^{\prime \prime}\right)\right)$ and $f\left(D S_{i}^{\Gamma}\left(R_{i}^{\prime}\right), D S_{-i}^{\Gamma}\left(R_{-i}^{\prime \prime}\right)\right)$ are each singletons for all $R_{-i}^{\prime \prime} \in \mathscr{R}_{-i}$, and since $R_{-i}^{\prime \prime} \in D S_{-i}^{\Gamma}\left(R_{-i}^{\prime \prime}\right)$ for all $R_{-i}^{\prime \prime} \in \mathscr{R}_{-i}$ by the revelation principle, we have $f\left(D S_{i}^{\Gamma}\left(R_{i}\right), R_{-i}^{\prime \prime}\right)=f\left(D S_{i}^{\Gamma}\left(R_{i}\right), D S_{-i}^{\Gamma}\left(R_{-i}^{\prime \prime}\right)\right)$ and $f\left(D S_{i}^{\Gamma}\left(R_{i}^{\prime}\right), R_{-i}^{\prime \prime}\right)=f\left(D S_{i}^{\Gamma}\left(R_{i}^{\prime}\right), D S_{-i}^{\Gamma}\left(R_{-i}^{\prime \prime}\right)\right)$ for all $R_{-i}^{\prime \prime} \in \mathscr{R}_{-i}$. Thus,

$$
f\left(D S_{i}^{\Gamma}\left(R_{i}\right), R_{-i}^{\prime \prime}\right) I_{i} f\left(D S_{i}^{\Gamma}\left(R_{i}^{\prime}\right), R_{-i}^{\prime \prime}\right) \text { for all } R_{-i}^{\prime \prime} \in \mathscr{R}_{-i} .
$$

Suppose $\hat{R}_{i} \in D S_{i}^{\Gamma}\left(R_{i}^{\prime}\right)$. Then, since $f\left(D S_{i}^{\Gamma}\left(R_{i}^{\prime}\right), R_{-i}^{\prime \prime}\right)$ is a singleton for all $R_{-i}^{\prime \prime} \in$ $\mathscr{R}_{-i}$, we have $f\left(\hat{R}_{i}, R_{-i}^{\prime \prime}\right)=f\left(D S_{i}^{\Gamma}\left(R_{i}^{\prime}\right), R_{-i}^{\prime \prime}\right)$ for all $R_{-i}^{\prime \prime} \in \mathscr{R}_{-i}$. So, it follows from (3) that $f\left(\hat{R}_{i}, R_{-i}^{\prime \prime}\right) I_{i} f\left(D S_{i}^{\Gamma}\left(R_{i}\right), R_{-i}^{\prime \prime}\right)$ for all $R_{-i}^{\prime \prime} \in \mathscr{R}_{-i}$. This implies $\hat{R}_{i} \in D S_{i}^{\Gamma}\left(R_{i}\right)$; thus $D S_{i}^{\Gamma}\left(R_{i}^{\prime}\right) \subseteq D S_{i}^{\Gamma}\left(R_{i}\right)$.

Since $D S_{i}^{\Gamma}\left(R_{i}^{\prime}\right) \subseteq D S_{i}^{\Gamma}\left(R_{i}\right)$, we have $f\left(D S_{i}^{\Gamma}\left(R_{i}^{\prime}\right), D S_{-i}^{\Gamma}\left(R_{-i}\right)\right) \subseteq f\left(D S_{i}^{\Gamma}\left(R_{i}\right), D S_{-i}^{\Gamma}\left(R_{-i}\right)\right)$. This implies

$$
f\left(D S_{i}^{\Gamma}\left(R_{i}^{\prime}\right), D S_{-i}^{\Gamma}\left(R_{-i}\right)\right)=f\left(D S_{i}^{\Gamma}\left(R_{i}\right), D S_{-i}^{\Gamma}\left(R_{-i}\right)\right),
$$

because $f\left(D S_{i}^{\Gamma}\left(R_{i}^{\prime}\right), D S_{-i}^{\Gamma}\left(R_{-i}\right)\right)$ and $f\left(D S_{i}^{\Gamma}\left(R_{i}\right), D S_{-i}^{\Gamma}\left(R_{-i}\right)\right)$ are singletons. Substituting (2) for $f$ in (4) produces $f\left(R_{i}^{\prime}, R_{-i}\right)=f(R)$.

Next, we look for sufficient conditions for dominant strategy implementation by the associated direct revelation mechanism. If a social choice function satisfies both strategy-proofness and quasi-strong-non-bossiness, then there is always a unique dominant strategy equilibrium outcome of the associated direct revelation mechanism (although there may exist some dominant strategy equilibria of the mechanism). Since the associated direct revelation mechanism has no undesired equilibrium outcomes, the mechanism can implement the social choice function in dominant strategy equilibria. ${ }^{8}$

\footnotetext{
${ }^{8}$ Laffont and Maskin (1982) proposed the notion of strictly truthful implementation in dominant strategy equilibria, which requires that the associated direct revelation mechanism should have no untruthful dominant strategy equilibria. Note that even if a social choice function satisfies strategy-proofness and quasi-strong-non-bossiness, it is not necessarily strictly truthfully implementable in dominant strategy equilibria, because there may be untruthful dominant strategy equilibria of the associated direct revelation mechanism.
} 
Theorem 2. If a social choice function $f$ satisfies both strategy-proofness and quasi-strong-non-bossiness, then it is dominant strategy implementable by the associated direct revelation mechanism in general environments.

Proof. Let $\Gamma:=(\mathscr{R}, f)$. Since $f$ satisfies strategy-proofness, $f(R) R_{i} f\left(R_{i}^{\prime}, R_{-i}\right)$ for all $R \in \mathscr{R}$, all $i \in N$, and all $R_{i}^{\prime} \in \mathscr{R}_{i}$. This implies $R_{i} \in D S_{i}^{\Gamma}\left(R_{i}\right)$ for all $i \in N$ and all $R_{i} \in \mathscr{R}_{i}$.

Pick any $R \in \mathscr{R}$ and any $i \in N$. Suppose $\hat{R}_{i} \in D S_{i}^{\Gamma}\left(R_{i}\right)$. Then, since $R_{i}, \hat{R}_{i} \in$ $D S_{i}^{\Gamma}\left(R_{i}\right)$, we have $f\left(R_{i}, R_{-i}^{\prime \prime}\right) R_{i} f\left(\hat{R}_{i}, R_{-i}^{\prime \prime}\right)$ and $f\left(\hat{R}_{i}, R_{-i}^{\prime \prime}\right) R_{i} f\left(R_{i}, R_{-i}^{\prime \prime}\right)$ for all $R_{-i}^{\prime \prime} \in$ $\mathscr{R}_{-i}$. This implies $f\left(R_{i}, R_{-i}^{\prime \prime}\right) I_{i} f\left(\hat{R}_{i}, R_{-i}^{\prime \prime}\right)$ for all $R_{-i}^{\prime \prime} \in \mathscr{R}_{-i}$. So, quasi-strongnon-bossiness implies $f(R)=f\left(\hat{R}_{i}, R_{-i}\right)$. Thus, we have $f(R)=f\left(D S_{i}^{\Gamma}\left(R_{i}\right), R_{-i}\right)$, where $f\left(D S_{i}^{\Gamma}\left(R_{i}\right), R_{-i}\right)=\left\{a \in A \mid a=f\left(R_{i}^{\prime}, R_{-i}\right)\right.$ for some $\left.R_{i}^{\prime} \in D S_{i}^{\Gamma}\left(R_{i}\right)\right\}$.

Next, consider $\left(\hat{R}_{i}, R_{-i}\right)$ such that $\hat{R}_{i} \in D S_{i}^{\Gamma}\left(R_{i}\right)$, and pick any $j \in N \backslash\{i\}$. Suppose $\hat{R}_{j} \in D S_{j}^{\Gamma}\left(R_{j}\right)$. Then, $f\left(R_{j}, R_{-j}^{\prime \prime}\right) R_{j} f\left(\hat{R}_{j}, R_{-j}^{\prime \prime}\right)$ and $f\left(\hat{R}_{j}, R_{-j}^{\prime \prime}\right) R_{j} f\left(R_{j}, R_{-j}^{\prime \prime}\right)$ for all $R_{-j}^{\prime \prime} \in \mathscr{R}_{-j}$, because $R_{j}, \hat{R}_{j} \in D S_{j}^{\Gamma}\left(R_{j}\right)$. So, $f\left(R_{j}, R_{-j}^{\prime \prime}\right) I_{j} f\left(\hat{R}_{j}, R_{-j}^{\prime \prime}\right)$ for all $R_{-j}^{\prime \prime} \in$ $\mathscr{R}_{-j}$. Hence, $f\left(\hat{R}_{i}, R_{-i}\right)=f\left(\hat{R}_{i}, \hat{R}_{j}, R_{-i, j}\right)$ by quasi-strong-non-bossiness. Thus, $f(R)=f\left(D S_{i}^{\Gamma}\left(R_{i}\right), R_{-i}\right)=f\left(D S_{i}^{\Gamma}\left(R_{i}\right), D S_{j}^{\Gamma}\left(R_{j}\right), R_{-i, j}\right)$, where $f\left(D S_{i}^{\Gamma}\left(R_{i}\right), D S_{j}^{\Gamma}\left(R_{j}\right), R_{-i, j}\right):=$ $\left\{a \in A \mid a=f\left(R_{i}^{\prime}, R_{j}^{\prime}, R_{-i, j}\right)\right.$ for some $R_{i}^{\prime} \in D S_{i}^{\Gamma}\left(R_{i}\right)$ and some $\left.R_{j}^{\prime} \in D S_{j}^{\Gamma}\left(R_{j}\right)\right\}$.

Iteration of these arguments for further agents in $N$ establishes that $f\left(D S E^{\Gamma}(R)\right)=$ $f(R)$. Since similar arguments hold for any $R \in \mathscr{R}$, we can conclude that $f\left(\operatorname{DSE}^{\Gamma}(R)\right)=$ $f(R)$ for any $R \in \mathscr{R}$.

The revelation principle tells us that if a social choice function is implementable in dominant strategy equilibria, then truth telling by each agent is a dominant strategy equilibrium of the associated direct revelation mechanism, i.e., the social choice function satisfies strategy-proofness. Therefore, when combined with the revelation principle, Theorems 1 and 2 lead to the following corollary. ${ }^{9}$

Corollary 1. A social choice function is dominant strategy implementable via the associated direct revelation mechanism in general environments if and only if it satisfies both strategy-proofness and quasi-strong-non-bossiness.

Quasi-strong-non-bossiness is automatically satisfied if indifference in preferences is not allowed. So, Corollary 1 implies that strategy-proofness is necessary and sufficient for dominant strategy implementation by the associated direct revelation mechanism in general environments unless preferences involve indifference.

\section{Implementation by an Indirect Mechanism}

In Section 3, we show that strategy-proofness and quasi-strong-non-bossiness are both necessary and sufficient for dominant strategy implementation via the

\footnotetext{
${ }^{9}$ Saijo et al. (2005) have independently obtained the same result.
} 
associated direct revelation mechanism. In this section, we demonstrate that it is possible to dominant strategy implement a social choice function that satisfies strategy-proofness but violates quasi-strong-non-bossiness, if an indirect mechanism is permitted to be used.

Example 1. Consider a two-agent, two-good pure exchange economy with initial endowment point $e=\left(\left(x_{1}^{1}, x_{1}^{2}\right),\left(x_{2}^{1}, x_{2}^{2}\right)\right)=((6,4),(4,6))$. Agents 1 and 2 have preferences $R_{1}, R_{1}^{\prime}$ and $R_{2}, R_{2}^{\prime}$, respectively, which are illustrated in Figure 1 .

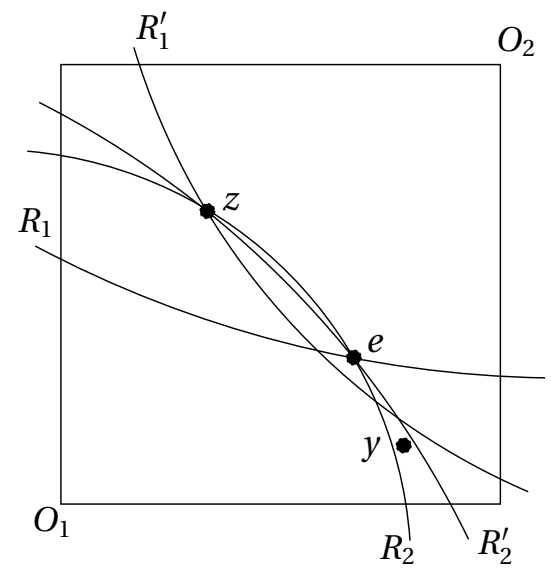

Figure 1: A Two-agent, Two-good Pure Exchange Economy

Consider an individually rational social choice function $f$ defined by

$$
f=\begin{array}{|c|c|}
R_{2} & R_{2}^{\prime} \\
\hline z & e \\
\hline e & R_{1} \\
\hline & R_{1}^{\prime}
\end{array}
$$

where $z=((4,6),(6,4))$. It is easy to check that $f$ satisfies strategy-proofness but fails to satisfy quasi-strong-non-bossiness. So, it follows from Corollary 1 that $f$ cannot be dominant strategy implemented by the associated direct revelation mechanism $\Gamma=\left(\left\{R_{1}, R_{1}^{\prime}\right\} \times\left\{R_{2}, R_{2}^{\prime}\right\}, f\right)$. In fact, $f\left(D S E^{\Gamma}\left(R_{1}, R_{2}\right)\right)=\{z, e\} \neq\{z\}=$ $f\left(R_{1}, R_{2}\right)$, since $\operatorname{DSE}^{\Gamma}\left(R_{1}, R_{2}\right)=\left\{\left(R_{1}, R_{2}\right),\left(R_{1}, R_{2}^{\prime}\right)\right\}$. However, $f$ can be dominant strategy implemented by an indirect mechanism $\Gamma^{\prime}=\left(\left\{m_{1}, m_{1}^{\prime}, m_{1}^{\prime \prime}\right\} \times\left\{m_{2}, m_{2}^{\prime}\right\}, g\right)$ such that

$$
g=\begin{array}{|c|c|c}
m_{2} & m_{2}^{\prime} & \\
\hline z & e & m_{1} \\
\cline { 1 - 2 } e & e & m_{1}^{\prime} \\
\cline { 1 - 2 } \boldsymbol{e} & y & m_{1}^{\prime \prime}
\end{array}
$$


where $y=((7.5,1.5),(2.5,8.5)) .{ }^{10}$ This is because $D S E^{\Gamma^{\prime}}\left(R_{1}, R_{2}\right)=\left(m_{1}, m_{2}\right), D S E^{\Gamma^{\prime}}\left(R_{1}, R_{2}^{\prime}\right)=$ $\left(m_{1}, m_{2}^{\prime}\right), D S E^{\Gamma^{\prime}}\left(R_{1}^{\prime}, R_{2}\right)=\left(m_{1}^{\prime}, m_{2}\right)$, and $D S E^{\Gamma^{\prime}}\left(R_{1}^{\prime}, R_{2}^{\prime}\right)=\left(m_{1}^{\prime}, m_{2}^{\prime}\right)$.

Example 1 illustrates that the existence of $y$ plays a key role in constructing $\Gamma^{\prime}$ that implements $f$ in dominant strategy equilibria. This indicates that it depends on the environment considered whether or not it is possible to construct an indirect mechanism that dominant strategy implements a given strategyproof social choice function. Hereafter, we focus on economic environments to study to what extent the use of indirect mechanisms expands the class of dominant strategy implementable social choice functions.

\section{Economic Environments}

In this section, we concentrate on weak separable environments, a weaker version of separable environments introduced by Jackson et al. (1994), and identify a condition that is sufficient for social choice functions to be implemented in dominant strategy equilibria. It should be noted that weak separable environments include as special cases pure exchange economies and economies with a transferable private good, because separable environments include such economies (see Jackson et al. (1994) or Jackson (2001)).

Before proceeding, let us introduce the definitions of weak separable environments and weakly dominated strategies. An environment is called a weak separable environment if the following three properties are satisfied.

Property 1 (Existence of a Common Worst Alternative). There exists $w \in A$ such that $a R_{i} w$ for all $i \in N$, all $R_{i} \in \mathscr{R}_{i}$, and all $a \in f(\mathscr{R})$, where $f(\mathscr{R}):=\{a \in A \mid a=f(R)$ for some $R \in \mathscr{R}\}$.

Property 2 (Weak Separability). For all $a \in A$, all $i \in N$, and all $R_{i} \in \mathscr{R}_{i}$, there exists $a^{-i} \in A$ such that $w R_{j}^{\prime} a^{-i}$ for all $j \in N \backslash\{i\}$ and all $R_{j}^{\prime} \in \mathscr{R}_{j}$, while $a I_{i} a^{-i}$.

Property 3 (Strict Value Distinction with a Reference Point $w$ ). For all $i \in N$ and all $R_{i}, R_{i}^{\prime} \in \mathscr{R}_{i}$, if $R_{i} \neq R_{i}^{\prime}$, then there exist $a, b \in A$ such that $a P_{i} b, b P_{i}^{\prime} a, a P_{i} w$, and $b P_{i}^{\prime} w$.

Note that Properties 1 and 2, respectively, are weaker than those introduced by Jackson et al. (1994) for separable environments, while Property 3 is the same as the one presented by Jackson et al. (1994).

A strategy $m_{i} \in M_{i}$ is a weakly dominated strategy of mechanism $(M, g)$ at $R_{i} \in \mathscr{R}_{i}$ if there exists a strategy $m_{i}^{\prime} \in M_{i}$ that weakly dominates $m_{i}$ at $R_{i} \in \mathscr{R}_{i}$ : $g\left(m_{i}^{\prime}, m_{-i}\right) R_{i} g\left(m_{i}, m_{-i}\right)$ for all $m_{-i} \in M_{-i}$ and $g\left(m_{i}^{\prime}, m_{-i}\right) P_{i} g\left(m_{i}, m_{-i}\right)$ for some $m_{-i} \in M_{-i}$.

We are now ready to state a main result regarding a sufficient condition for dominant strategy implementation in weak separable environments.

\footnotetext{
${ }^{10}$ This type of indirect mechanism is called an augmented revelation mechanism (see Mookherjee and Reichelstein (1990)).
} 
Theorem 3. If a social choice function $f$ satisfies strategy-proofness, then it is dominant strategy implementable in weak separable environments.

Proof. Fix an arbitrary $w \in A$ that satisfies Property 1 . Given $a \in A, i \in N$, and $R_{i} \in \mathscr{R}_{i}$, let $[a]_{R_{i}}$ denote an arbitrary alternative in $A$ such that $a I_{i}[a]_{R_{i}}$ and $w R_{j}^{\prime}[a]_{R_{i}}$ for all $j \in N \backslash\{i\}$ and all $R_{j}^{\prime} \in \mathscr{R}_{j}$. Note that Property 2 guarantees the existence of $[a]_{R_{i}}$ for any $a \in A$, any $i \in N$, and any $R_{i} \in \mathscr{R}_{i}$.

Now we construct an augmented revelation mechanism $\Gamma=(M, g)$, by using ideas similar to those of the mechanism constructed by Jackson et al. (1994) for undominated Nash implementation. Agent $i$ 's message space is

$$
M_{i}:=\mathscr{R}_{i} \cup(A \times A) .
$$

That is, each agent announces either own preferences or a pair of alternatives. A typical message for agent $i \in N$ is denoted by $m_{i}=R_{i}^{i}$ or $\left(a^{i}, b^{i}\right)$. The outcome function $g$ is defined as follows.

Rule 1: If $m_{i}=R_{i}^{i} \in \mathscr{R}_{i}$ for all $i \in N$, then $g(m):=f\left(R_{1}^{1}, R_{2}^{2}, \ldots, R_{n}^{n}\right)$. Note that $g(m) R_{i}^{i} w$ for all $i \in N$ by Property 1 .

Rule 2: If $m_{i}=R_{i}^{i} \in \mathscr{R}_{i}$ for some $i \in N$ and $m_{j}=\left(a^{j}, b^{j}\right) \in(A \times A)$ for any $j \neq i$, then $g(m):=\left[\arg _{\max } \max _{B\}} R_{i}^{i}\right]_{R_{i}^{i}}$, where $B:=\left\{a^{j}, b^{j} \in A \mid j \neq i\right\}$. Ties are broken in some arbitrary manner unless $\arg \max _{B \cup\{w\}} R_{i}^{i}$ is a singleton. Note that $g(m) R_{i}^{i} w$ and $w R_{j}^{\prime} g(m)$ for all $j \neq i$ and all $R_{j}^{\prime} \in \mathscr{R}_{j}$ by construction.

Rule 3: In all other cases, $g(m):=w$.

We want to show that $D S_{i}^{\Gamma}\left(R_{i}\right)=\left\{R_{i}\right\}$ for all $i \in N$ and all $R_{i} \in \mathscr{R}_{i}$.

Step 1: For each agent, announcing a pair of alternatives is always weakly dominated by the truthful revelation of own preferences.

Pick any $i \in N$. Suppose that her true preference relation is $R_{i}^{*} \in \mathscr{R}_{i}$. Let $m_{i} \in M_{i}$ be such that $m_{i}=\left(a^{i}, b^{i}\right) \in(A \times A)$, and $m_{i}^{\prime} \in M_{i}$ be such that $m_{i}^{\prime}=R_{i}^{*}$.

Case 1-1: $n \geq 3$.

Subcase 1: $m_{j}=R_{j}^{j} \in \mathscr{R}_{j}$ for all $j \neq i$.

We have $g\left(m_{i}, m_{-i}\right)=w$ and $g\left(m_{i}^{\prime}, m_{-i}\right)=f\left(R_{i}^{*}, R_{-i}^{-i}\right)$ by Rules 3 and 1 , respectively. Since $f\left(R_{i}^{*}, R_{-i}^{-i}\right) R_{i}^{*} w$ by Property $1, g\left(m_{i}^{\prime}, m_{-i}\right) R_{i}^{*} g\left(m_{i}, m_{-i}\right)$.

Subcase 2: $m_{j}=\left(a^{j}, b^{j}\right) \in(A \times A)$ for all $j \neq i$.

We have $g\left(m_{i}, m_{-i}\right)=w$ by Rule 3 , whereas $g\left(m_{i}^{\prime}, m_{-i}\right)=\left[\operatorname{argmax}_{B \cup\{w\}} R_{i}^{*}\right]_{R_{i}^{*}}$ by Rule 2, where $B=\left\{a^{j}, b^{j} \in A \mid j \neq i\right\}$. Since $\left[\arg \max _{B \cup\{w\}} R_{i}^{*}\right]_{R_{i}^{*}} R_{i}^{*} w$, we obtain $g\left(m_{i}^{\prime}, m_{-i}\right) R_{i}^{*} g\left(m_{i}, m_{-i}\right)$. In particular, if $m_{j}=(a, a)$ for all $j \neq i$, and if $a P_{i}^{*} w,{ }^{11}$ then $g\left(m_{i}^{\prime}, m_{-i}\right) P_{i}^{*} g\left(m_{i}, m_{-i}\right)$.

Subcase 3: $m_{j}=R_{j}^{j} \in \mathscr{R}_{j}$ for some $j \neq i$ and $m_{k}=\left(a^{k}, b^{k}\right) \in(A \times A)$ for all $k \in$ $N \backslash\{i, j\}$.

\footnotetext{
${ }^{11}$ Property 3 assures that there exists $a \in A$ such that $a P_{i}^{*} w$.
} 
It follows from Rule 2 that $g\left(m_{i}, m_{-i}\right)=\left[\operatorname{argmax}_{B \cup\{w\}} R_{j}^{j}\right]_{R_{j}^{j}}$, where $B=\left\{a^{h}, b^{h} \in A \mid h \neq j\right\}$.

On the other hand, $g\left(m_{i}^{\prime}, m_{-i}\right)=w$ by Rule 3. Since $w R_{i}^{*}\left[\arg _{B} \max _{B \cup\{w\}} R_{j}^{j}\right]_{R_{j}^{j}}$, we have $g\left(m_{i}^{\prime}, m_{-i}\right) R_{i}^{*} g\left(m_{i}, m_{-i}\right)$.

Subcase 4: All other cases.

Rule 3 implies $g\left(m_{i}, m_{-i}\right)=w$ and $g\left(m_{i}^{\prime}, m_{-i}\right)=w$, implying $g\left(m_{i}^{\prime}, m_{-i}\right) R_{i}^{*} g\left(m_{i}, m_{-i}\right)$.

Case 1-2: $n=2$.

Subcase 1: $m_{j}=R_{j}^{j} \in \mathscr{R}_{j}$ for $j \neq i$.

Rule 2 implies $g\left(m_{i}, m_{j}\right)=\left[\operatorname{argmax}_{B \cup\{w\}} R_{j}^{j}\right]_{R_{j}^{j}}$ where $B=\left\{a^{i}, b^{i}\right\}$, while Rule 1 implies $g\left(m_{i}^{\prime}, m_{j}\right)=f\left(R_{i}^{*}, R_{j}^{j}\right)$. Since $f\left(R_{i}^{*}, R_{j}^{j}\right) R_{i}^{*} w$ by Property 1 , and since $w R_{i}^{*}\left[\arg \max _{B \cup\{w\}} R_{j}^{j}\right]_{R_{j}^{j}}$, we obtain $g\left(m_{i}^{\prime}, m_{j}\right) R_{i}^{*} g\left(m_{i}, m_{j}\right)$ by transitivity.

Subcase 2: $m_{j}=\left(a^{j}, b^{j}\right) \in(A \times A)$ for $j \neq i$.

See Case 1-1 Subcase 2.

The above cases together establish that $m_{i}$ is weakly dominated by $m_{i}^{\prime}$ at $R_{i}^{*}$.

Step 2: For every agent, misrepresenting own preferences is always weakly dominated by the truthful announcement of own preferences.

Pick any $i \in N$. Suppose that her true preference relation is $R_{i}^{*} \in \mathscr{R}_{i}$. Let $m_{i} \in M_{i}$ be such that $m_{i}=R_{i}^{i} \in \mathscr{R}_{i} \backslash\left\{R_{i}^{*}\right\}$, and $m_{i}^{\prime} \in M_{i}$ be such that $m_{i}^{\prime}=R_{i}^{*}$.

Case 2-1: $m_{j}=R_{j}^{j} \in \mathscr{R}_{j}$ for all $j \neq i$.

Rule 1 implies $g\left(m_{i}, m_{-i}\right)=f\left(R_{i}^{i}, R_{-i}^{-i}\right)$ and $g\left(m_{i}^{\prime}, m_{-i}\right)=f\left(R_{i}^{*}, R_{-i}^{-i}\right)$. Since strategyproofness implies $f\left(R_{i}^{*}, R_{-i}^{-i}\right) R_{i}^{*} f\left(R_{i}^{i}, R_{-i}^{-i}\right)$, we obtain $g\left(m_{i}^{\prime}, m_{-i}\right) R_{i}^{*} g\left(m_{i}, m_{-i}\right)$.

Case 2-2: $m_{j}=\left(a^{j}, b^{j}\right) \in(A \times A)$ for all $j \neq i$.

It follows from Rule 2 that $g\left(m_{i}, m_{-i}\right)=\left[\arg \max _{B \cup\{w\}} R_{i}^{i}\right]_{R_{i}^{i}}$ and $g\left(m_{i}^{\prime}, m_{-i}\right)=$ $\left[\arg \max _{B \cup\{w\}} R_{i}^{*}\right]_{R_{i}^{*}}$, where $B=\left\{a^{j}, b^{j} \in A \mid j \neq i\right\}$. Since agent $i$ 's true preference relation is now $R_{i}^{*}$, $\left[\operatorname{argmax}_{B \cup\{w\}} R_{i}^{*}\right]_{R_{i}^{*}} R_{i}^{*}\left[\operatorname{argmax}_{B \cup\{w\}} R_{i}^{i}\right]_{R_{i}^{i}}$. So, we have $g\left(m_{i}^{\prime}, m_{-i}\right) R_{i}^{*} g\left(m_{i}, m_{-i}\right)$. Suppose in turn that agent $i$ 's true preference relation is $R_{i}^{i}$. Then, since $\left[\arg \max _{B \cup\{w\}} R_{i}^{i}\right]_{R_{i}^{i}} R_{i}^{i}\left[\arg \max _{B \cup\{w\}} R_{i}^{*}\right]_{R_{i}^{*}}$, we obtain $g\left(m_{i}, m_{-i}\right) R_{i}^{i} g\left(m_{i}^{\prime}, m_{-i}\right)$. Especially, if $m_{j}=(a, b)$ for all $j \neq i$, and if $a P_{i}^{*} b, b P_{i}^{i} a$, $a P_{i}^{*} w$, and $b P_{i}^{i} w{ }^{12}$ then $g\left(m_{i}^{\prime}, m_{-i}\right) P_{i}^{*} g\left(m_{i}, m_{-i}\right)$ and $g\left(m_{i}, m_{-i}\right) P_{i}^{i} g\left(m_{i}^{\prime}, m_{-i}\right)$.

Case 2-3: All other cases.

By Rule 3, $g\left(m_{i}, m_{-i}\right)=w$ and $g\left(m_{i}^{\prime}, m_{-i}\right)=w$, which implies $g\left(m_{i}^{\prime}, m_{-i}\right) R_{i}^{*} g\left(m_{i}, m_{-i}\right)$.

The above cases together establish that $m_{i}$ is weakly dominated by $m_{i}^{\prime}$ at $R_{i}^{*}$.

Steps 1 and 2 together imply that, for each agent, the truthful revelation of own preferences always weakly dominates both announcements of a pair of alternatives and false reports of own preferences. Thus, we establish that

\footnotetext{
${ }^{12}$ Property 3 ensures that there exist $a, b \in A$ such that $a P_{i}^{*} b, b P_{i}^{i} a, a P_{i}^{*} w$, and $b P_{i}^{i} w$.
} 
$D S_{i}^{\Gamma}\left(R_{i}\right)=\left\{R_{i}\right\}$ for all $i \in N$ and all $R_{i} \in \mathscr{R}_{i}$; i.e., $D S E^{\Gamma}(R)=\{R\}$ for all $R \in \mathscr{R}$. Therefore, we can conclude from Rule 1 that $g\left(D S E^{\Gamma}(R)\right)=f(R)$ for all $R \in \mathscr{R}$.

Note that our mechanism works not only when $n \geq 3$ but also when $n=2$, as the mechanism of Jackson et al. (1994) works. Note also that our mechanism uses not only Property 1 but also Property 2 to punish agents who do not announce their truthful preferences.

The following corollary follows immediately from the revelation principle and Theorem 3.

Corollary 2. A social choice function is dominant strategy implementable in weak separable environments if and only if it satisfies strategy-proofness.

Corollary 2 tells us that all strategy-proof social choice functions are implementable in dominant strategy equilibria if indirect mechanisms are allowed to be used. This is in stark contrast to Corollary 1, which states that only strategyproof and quasi-strong-non-bossy social choice functions are dominant strategy implementable if the associated direct revelation mechanisms are used.

Our mechanism appears to be similar to the one devised by Jackson et al. (1994) for undominated Nash implementation in separable environments. It is true that their mechanism as well as the one constructed by Sjöström (1994) can dominant strategy implement almost all strategy-proof social choice functions in weak separable environments. But, an important difference is that our mechanism can dominant strategy implement the strategy-proof social choice function that assigns a common worst alternative to some preference profile, whereas their mechanisms cannot. ${ }^{13}$ Thus, our mechanism has the advantage of being able to dominant strategy implement every strategy-proof social choice function (although the advantage is somewhat limited because our mechanism is not necessarily useful for undominated Nash implementation).

We conclude this section with a brief discussion of implementation in another equilibrium concept. It is easy to check that our mechanism is bounded, and that in weak separable environments, our bounded mechanism can implement every strategy-proof social choice function even in undominated strategies. So, Theorem 3 implies the following theorem, when coupled with Corollary 1 in Jackson (1992) which says that strategy-proofness is necessary for bounded implementation in undominated strategies.

Theorem 4. A social choice function is implementable in undominated strategies by a bounded mechanism in weak separable environments if and only if it satisfies strategy-proofness.

Theorem 4 indicates that in weak separable environments, implementation in dominant strategy equilibria is equivalent to bounded implementation in un-

\footnotetext{
${ }^{13}$ The difference stems from the fact that Jackson et al. (1994) and Sjöström (1994) needed a slightly stronger property than Property 1.
} 
dominated strategies, in the sense that the set of social choice functions implementable in dominant strategy equilibria coincides with that of social choice functions boundedly implementable in undominated strategies.

\section{Pure Exchange Economies}

In this section, we explore a condition which is sufficient for dominant strategy implementation in pure exchange economies.

Now let us introduce some notation and two kinds of definitions of $l$-good pure exchange economies. Let $a_{i} \in \mathbb{R}_{+}^{l}$ and $e_{i} \in \mathbb{R}_{+}^{l}$ denote agent $i$ 's consumption bundle and initial endowment, respectively. The set of consumption bundles is $C:=\left\{a_{i} \in \mathbb{R}_{+}^{l} \mid a_{i} \leq \sum_{i \in N} e_{i}\right\}$. In this section, we assume that each agent has preferences over $C \subseteq \mathbb{R}_{+}^{l}$. Let $\mathscr{R}_{i}^{E}$ be agent $i$ 's set of preferences: $R_{i} \in \mathscr{R}_{i}^{E}$ only if $R_{i}$ is complete, transitive, and monotone. Let $A^{E}:=\left\{\left(a_{1}, a_{2}, \ldots, a_{n}\right) \in \mathbb{R}_{+}^{l n} \mid \sum_{i \in N} a_{i} \leq \sum_{i \in N} e_{i}\right\}$ and $A^{E^{B}}:=\left\{\left(a_{1}, a_{2}, \ldots, a_{n}\right) \in \mathbb{R}_{+}^{l n} \mid \sum_{i \in N} a_{i}=\sum_{i \in N} e_{i}\right\}$. A pure exchange economy is the environment $\left(N, A^{E}, \mathscr{R}^{E}\right)$, and a pure exchange economy without free disposal is the environment $\left(N, A^{E^{B}}, \mathscr{R}^{E}\right)$.

We begin by identifying a necessary and sufficient condition for dominant strategy implementation in pure exchange economies. Since Properties 1 and 2 are satisfied in pure exchange economies by setting $w=(0,0, \ldots, 0) \in A^{E}$ for any social choice function, Corollary 3 below follows immediately from Corollary 2 .

Corollary 3. Suppose that Property 3 is satisfied. ${ }^{14}$ Then, a social choice function is dominant strategy implementable in pure exchange economies if and only if it satisfies strategy-proofness.

We turn next to a sufficient condition for dominant strategy implementation in pure exchange economies without free disposal. Contrary to the case of pure exchange economies, Properties 1 and 2 do not necessarily hold in pure exchange economies without free disposal, partly because it is impossible to choose $w \in A^{E^{B}}$ for some social choice functions. So, Theorem 3 leaves open the question of what condition is sufficient for dominant strategy implementation in pure exchange economies without free disposal. Theorem 5 below provides a partial answer to this question.

Property 4 (Strict Value Distinction). For all $i \in N$ and all $R_{i}, R_{i}^{\prime} \in \mathscr{R}_{i}$, if $R_{i} \neq R_{i}^{\prime}$, then there exist $a, b \in A$ such that $a P_{i} b$ and $b P_{i}^{\prime} a$.

Theorem 5. Suppose that $n \geq 3$ and Property 4 is satisfied. Then, if a social choice function $f$ satisfies strategy-proofness, then it is dominant strategy implementable in pure exchange economies without free disposal.

\footnotetext{
${ }^{14}$ In pure exchange economies, Property 3 is equivalent to Property 4 defined below by setting $w=(0,0, \ldots, 0) \in A^{E}$.
} 
Proof. We construct an augmented revelation mechanism $\Gamma=(M, g)$ in the following way. The message space for agent $i \in N$ is

$$
M_{i}:=\mathscr{R}_{i}^{E} \cup(C \times C) .
$$

That is, each agent reports either own preferences or a pair of consumption bundles. A typical message for agent $i \in N$ is denoted by $m_{i}=R_{i}^{i}$ or $\left(c^{i}, d^{i}\right)$. Given $m \in M$, let $S(m):=\left\{i \in N \mid m_{i} \in \mathscr{R}_{i}^{E}\right\}$ and $S^{c}(m):=\left\{i \in N \mid m_{i} \in(C \times C)\right\}$. Note that $S(m) \cap S^{c}(m)=\varnothing$ and $S(m) \cup S^{c}(m)=N$ for every $m \in M$. Given $m \in M$, let

$$
\hat{\imath}(m):= \begin{cases}n & \text { if } 1, n \in S(m) \\ \min S(m) & \text { otherwise }\end{cases}
$$

Let $f_{i}$ and $g_{i}$ denote agent $i$ 's consumption bundle assigned by $f$ and $g$, respectively. The outcome function $g$ is as follows.

Rule 1: If $\# S(m)=n$ and $\# S^{c}(m)=0$, then $g_{i}(m):=f_{i}\left(R_{1}^{1}, R_{2}^{2}, \ldots, R_{n}^{n}\right)$ for all $i \in N$. Rule $q$ for $q=2,3, \ldots, n-1$ : If $\# S(m)=n-q+1$ and $\# S^{c}(m)=q-1$, then

$$
\begin{aligned}
g_{\hat{\imath}(m)}(m) & :=\underset{D}{\arg \max _{D} R_{\hat{\imath}(m)}^{\hat{\imath}(m)},} \\
g_{j}(m) & :=\frac{\sum_{h \in N} e_{h}-g_{\hat{\imath}(m)}(m)}{\# S(m)-1} \text { for all } j \in S(m) \backslash\{\hat{\imath}(m)\}, \text { and } \\
g_{k}(m) & :=0 \text { for all } k \in S^{c}(m),
\end{aligned}
$$

where $D:=\left\{c^{k}, d^{k} \in C \mid k \in S^{c}(m)\right\}$. Unless $\arg \max _{D} R_{\hat{\imath}(m)}^{\hat{\imath}(m)}$ is a singleton, ties are broken in a lexicographic way (e.g., in a way where a good with a lower index has a higher priority). Notice that for all $i \in N$, there exists $m \in M$ with $\# S(m)=2$ such that $\hat{\imath}(m)=i$.

Rule $n$ : If $\# S(m)=1$ and $\# S^{c}(m)=n-1$, then

$$
g_{i}(m):=\sum_{h \in N} e_{h} \text { for } i \in S(m) \quad \text { and } \quad g_{j}(m):=0 \text { for all } j \in S^{c}(m) .
$$

Rule $n+1$ : If $\# S(m)=0$ and $\# S^{c}(m)=n$, then $g_{i}(m)=e_{i}$ for all $i \in N$.

We want to show that $D S_{i}^{\Gamma}\left(R_{i}\right)=\left\{R_{i}\right\}$ for every $i \in N$ and every $R_{i} \in \mathscr{R}_{i}^{E}$.

Step 1: For each agent, reporting a pair of consumption bundles is always weakly dominated by the truthful announcement of own preferences.

Pick any $i \in N$. Suppose that her true preference relation is $R_{i}^{*} \in \mathscr{R}_{i}^{E}$. Let $m_{i} \in M_{i}$ be such that $m_{i}=\left(c^{i}, d^{i}\right) \in(C \times C)$, and $m_{i}^{\prime} \in M_{i}$ be such that $m_{i}^{\prime}=R_{i}^{*}$.

Case 1-1: $m_{j}=R_{j}^{j} \in \mathscr{R}_{j}^{E}$ for all $j \neq i$.

Rules 2 and 1 imply that $g_{i}\left(m_{i}, m_{-i}\right)=0$ and $g_{i}\left(m_{i}^{\prime}, m_{-i}\right)=f_{i}\left(R_{i}^{*}, R_{-i}^{-i}\right)$, respectively. So, we have $g_{i}\left(m_{i}^{\prime}, m_{-i}\right) R_{i}^{*} g_{i}\left(m_{i}, m_{-i}\right)$, because $f_{i}\left(R_{i}^{*}, R_{-i}^{-i}\right) \geq 0$.

Case 1-2: $m_{j}=\left(c^{j}, d^{j}\right) \in(C \times C)$ for all $j \neq i$. 
We have $g_{i}\left(m_{i}, m_{-i}\right)=e_{i}$ and $g_{i}\left(m_{i}^{\prime}, m_{-i}\right)=\sum_{h \in N} e_{h}$ by Rules $n+1$ and $n$. Since $\sum_{h \in N} e_{h} \geq e_{i}$, we have $g_{i}\left(m_{i}^{\prime}, m_{-i}\right) R_{i}^{*} g_{i}\left(m_{i}, m_{-i}\right)$.

Case 1-3: $m_{j}=R_{j}^{j} \in \mathscr{R}_{j}^{E}$ for some $j \neq i$ and $m_{k}=\left(c^{k}, d^{k}\right) \in(C \times C)$ for all $k \in N \backslash\{i, j\}$.

Subcase 1: $\hat{\imath}\left(m_{i}^{\prime}, m_{-i}\right)=i$.

Rules $n$ and $n-1$, respectively, imply that $g_{i}\left(m_{i}, m_{-i}\right)=0$ and $g_{i}\left(m_{i}^{\prime}, m_{-i}\right)=$ $\arg \max _{D} R_{i}^{*}$, where $D=\left\{c^{h}, d^{h} \in C \mid h \in S^{c}\left(m_{i}^{\prime}, m_{-i}\right)\right\}$. Since $\operatorname{argmax}_{D} R_{i}^{*} \geq 0$, it holds that $g_{i}\left(m_{i}^{\prime}, m_{-i}\right) R_{i}^{*} g_{i}\left(m_{i}, m_{-i}\right)$. In particular, $g_{i}\left(m_{i}^{\prime}, m_{-i}\right) P_{i}^{*} g_{i}\left(m_{i}, m_{-i}\right)$ if $m_{k}=\left(\sum_{h \in N} e_{h}, \sum_{h \in N} e_{h}\right)$ for some $k \in N \backslash\{i, j\}$.

Subcase 2: $\hat{\imath}\left(m_{i}^{\prime}, m_{-i}\right)=j$.

We have $g_{i}\left(m_{i}, m_{-i}\right)=0$ by Rule $n$, while $g_{i}\left(m_{i}^{\prime}, m_{-i}\right)=\sum_{h \in N} e_{h}-g_{j}\left(m_{i}^{\prime}, m_{-i}\right)$ by Rule $n-1$. Since $g_{j}\left(m_{i}^{\prime}, m_{-i}\right)=\arg \max _{D} R_{j}^{j}$, where $D=\left\{c^{h}, d^{h} \in C \mid h \in S^{c}\left(m_{i}^{\prime}, m_{-i}\right)\right\}$, we obtain $\sum_{h \in N} e_{h}-g_{j}\left(m_{i}^{\prime}, m_{-i}\right) \geq 0$. Hence, $g_{i}\left(m_{i}^{\prime}, m_{-i}\right) R_{i}^{*} g_{i}\left(m_{i}, m_{-i}\right)$.

Case 1-4: All other cases.

Assume without loss of generality that $\# S\left(m_{i}^{\prime}, m_{-i}\right)=n-q+1$ and $\# S^{c}\left(m_{i}^{\prime}, m_{-i}\right)=$ $q-1$ for some $q=2,3, \ldots, n-2$.

Subcase 1: $\hat{\imath}\left(m_{i}^{\prime}, m_{-i}\right)=i$.

We obtain $g_{i}\left(m_{i}, m_{-i}\right)=0$ and $g_{i}\left(m_{i}^{\prime}, m_{-i}\right)=\arg \max _{D} R_{i}^{*}$ by Rules $q+1$ and $q$ respectively, where $D=\left\{c^{h}, d^{h} \in C \mid h \in S^{c}\left(m_{i}^{\prime}, m_{-i}\right)\right\}$. So, we have $g_{i}\left(m_{i}^{\prime}, m_{-i}\right) R_{i}^{*} g_{i}\left(m_{i}, m_{-i}\right)$, because $\arg \max _{D} R_{i}^{*} \geq 0$.

Subcase 2: $\hat{\imath}\left(m_{i}^{\prime}, m_{-i}\right)=j$ for some $j \in S\left(m_{i}^{\prime}, m_{-i}\right) \backslash\{i\}$.

Rule $q+1$ implies $g_{i}\left(m_{i}, m_{-i}\right)=0$, whereas Rule $q$ implies $g_{i}\left(m_{i}^{\prime}, m_{-i}\right)=\frac{\sum_{h \in N} e_{h}-g_{j}\left(m_{i}^{\prime}, m_{-i}\right)}{\# S\left(m_{i}^{\prime}, m_{-i}\right)-1}$.

Since $g_{j}\left(m_{i}^{\prime}, m_{-i}\right)=\arg \max _{D} R_{j}^{j}$, where $D=\left\{c^{h}, d^{h} \in C \mid h \in S^{c}\left(m_{i}^{\prime}, m_{-i}\right)\right\}$, it holds that $\frac{\sum_{h \in N} e_{h}-g_{j}\left(m_{i}^{\prime}, m_{-i}\right)}{\# S\left(m_{i}^{\prime}, m_{-i}\right)-1} \geq 0$. So, $g_{i}\left(m_{i}^{\prime}, m_{-i}\right) R_{i}^{*} g_{i}\left(m_{i}, m_{-i}\right)$.

The above cases together establish that $m_{i}$ is weakly dominated by $m_{i}^{\prime}$ at $R_{i}^{*}$.

Step 2: For every agent, falsely reporting own preferences is always weakly dominated by truthfully reporting own preferences.

Pick any $i \in N$. Suppose that her true preference relation is $R_{i}^{*} \in \mathscr{R}_{i}^{E}$. Let $m_{i} \in M_{i}$ be such that $m_{i}=R_{i}^{i} \in \mathscr{R}_{i}^{E} \backslash\left\{R_{i}^{*}\right\}$, and $m_{i}^{\prime} \in M_{i}$ be such that $m_{i}^{\prime}=R_{i}^{*}$.

Case 2-1: $m_{j}=R_{j}^{j} \in \mathscr{R}_{j}^{E}$ for all $j \neq i$.

By Rule $1, g_{i}\left(m_{i}, m_{-i}\right)=f_{i}\left(R_{i}^{i}, R_{-i}^{-i}\right)$ and $g_{i}\left(m_{i}^{\prime}, m_{-i}\right)=f_{i}\left(R_{i}^{*}, R_{-i}^{-i}\right)$. This implies $g_{i}\left(m_{i}^{\prime}, m_{-i}\right) R_{i}^{*} g_{i}\left(m_{i}, m_{-i}\right)$, because $f_{i}\left(R_{i}^{*}, R_{-i}^{-i}\right) R_{i}^{*} f_{i}\left(R_{i}^{i}, R_{-i}^{-i}\right)$ by strategy-proofness.

Case 2-2: $m_{j}=\left(c^{j}, d^{j}\right) \in(C \times C)$ for all $j \neq i$.

Rule $n$ implies $g_{i}\left(m_{i}, m_{-i}\right)=\sum_{h \in N} e_{h}$ and $g_{i}\left(m_{i}^{\prime}, m_{-i}\right)=\sum_{h \in N} e_{h}$, which implies $g_{i}\left(m_{i}^{\prime}, m_{-i}\right) R_{i}^{*} g_{i}\left(m_{i}, m_{-i}\right)$.

Case 2-3: All other cases.

Assume without loss of generality that $\# S\left(m_{i}^{\prime}, m_{-i}\right)=n-q+1$ and $\# S^{c}\left(m_{i}^{\prime}, m_{-i}\right)=$ $q-1$ for some $q=2,3, \ldots, n-1$. Note that $\hat{\imath}\left(m_{i}^{\prime}, m_{-i}\right)=\hat{\imath}\left(m_{i}, m_{-i}\right)$, because $S\left(m_{i}^{\prime}, m_{-i}\right)=S\left(m_{i}, m_{-i}\right)$. 
Subcase 1: $\hat{\imath}\left(m_{i}^{\prime}, m_{-i}\right)=i$.

Rule $q$ implies $g_{i}\left(m_{i}, m_{-i}\right)=\arg \max _{D} R_{i}^{i}$ and $g_{i}\left(m_{i}^{\prime}, m_{-i}\right)=\operatorname{argmax}_{D^{\prime}} R_{i}^{*}$, where $D=\left\{c^{h}, d^{h} \in C \mid h \in S^{c}\left(m_{i}, m_{-i}\right)\right\}$ and $D^{\prime}=\left\{c^{h}, d^{h} \in C \mid h \in S^{c}\left(m_{i}^{\prime}, m_{-i}\right)\right\}$. Notice that $D=D^{\prime}$, because $S^{c}\left(m_{i}, m_{-i}\right)=S^{c}\left(m_{i}^{\prime}, m_{-i}\right)$. So, since agent $i$ 's true preference relation is now $R_{i}^{*}$, we have $\left(\operatorname{argmax}_{D^{\prime}} R_{i}^{*}\right) R_{i}^{*}\left(\arg _{\max } R_{i}^{i}\right)$. This implies $g_{i}\left(m_{i}^{\prime}, m_{-i}\right) R_{i}^{*} g_{i}\left(m_{i}, m_{-i}\right)$. Suppose in turn that her true preference relation is $R_{i}^{i}$. Then, $\left(\arg \max _{D} R_{i}^{i}\right) R_{i}^{i}\left(\arg _{\max _{D^{\prime}}} R_{i}^{*}\right)$, implying $g_{i}\left(m_{i}, m_{-i}\right) R_{i}^{i} g_{i}\left(m_{i}^{\prime}, m_{-i}\right)$. Moreover, $g_{i}\left(m_{i}^{\prime}, m_{-i}\right) P_{i}^{*} g_{i}\left(m_{i}, m_{-i}\right)$ and $g_{i}\left(m_{i}, m_{-i}\right) P_{i}^{i} g_{i}\left(m_{i}^{\prime}, m_{-i}\right)$, provided that $m_{h}=(c, d)$ for all $h \in S^{c}\left(m_{i}, m_{-i}\right)=S^{c}\left(m_{i}^{\prime}, m_{-i}\right)$ and that $c P_{i}^{*} d$ and $d P_{i}^{i} c{ }^{15}$

Subcase 2: $\hat{\imath}\left(m_{i}^{\prime}, m_{-i}\right)=j$ for some $j \in S\left(m_{i}^{\prime}, m_{-i}\right) \backslash\{i\}$.

It follows from Rule $q$ that $g_{j}\left(m_{i}, m_{-i}\right)=\arg \max _{D} R_{j}^{j}$ and $g_{j}\left(m_{i}^{\prime}, m_{-i}\right)=\arg \max _{D^{\prime}} R_{j}^{j}$, where $D=\left\{c^{h}, d^{h} \in C \mid h \in S^{c}\left(m_{i}, m_{-i}\right)\right\}$ and $D^{\prime}=\left\{c^{h}, d^{h} \in C \mid h \in S^{c}\left(m_{i}^{\prime}, m_{-i}\right)\right\}$. Note that $D=D^{\prime}$, since $S^{c}\left(m_{i}, m_{-i}\right)=S^{c}\left(m_{i}^{\prime}, m_{-i}\right)$. These imply $g_{j}\left(m_{i}, m_{-i}\right)=$ $g_{j}\left(m_{i}^{\prime}, m_{-i}\right){ }^{16}$ Hence, together with the fact that \#S( $\left.m_{i}, m_{-i}\right)=\# S\left(m_{i}^{\prime}, m_{-i}\right)$, it follows from Rule $q$ that $g_{i}\left(m_{i}, m_{-i}\right)=\frac{\sum_{h \in N} e_{h}-g_{j}\left(m_{i}, m_{-i}\right)}{\# S\left(m_{i}, m_{-i}\right)-1}=\frac{\sum_{h \in N} e_{h}-g_{j}\left(m_{i}^{\prime}, m_{-i}\right)}{\# S\left(m_{i}^{\prime}, m_{-i}\right)-1}=$ $g_{i}\left(m_{i}^{\prime}, m_{-i}\right)$. This implies $g_{i}\left(m_{i}^{\prime}, m_{-i}\right) R_{i}^{*} g_{i}\left(m_{i}, m_{-i}\right)$.

The above cases together establish that $m_{i}$ is weakly dominated by $m_{i}^{\prime}$ at $R_{i}^{*}$.

Steps 1 and 2 together imply that, for every agent, truthfully reporting own preferences always weakly dominates both announcing a pair of consumption bundles and misrepresenting own preferences. Thus, we establish that $D S_{i}^{\Gamma}\left(R_{i}\right)=$ $\left\{R_{i}\right\}$ for all $i \in N$ and all $R_{i} \in \mathscr{R}_{i}^{E}$; i.e., $D S E^{\Gamma}(R)=\{R\}$ for all $R \in \mathscr{R}^{E}$. Therefore, we can conclude from Rule 1 that $g\left(D S E^{\Gamma}(R)\right)=f(R)$ for all $R \in \mathscr{R}^{E}$.

It should be noted that if there are only two agents, our mechanism used to prove Theorem 5 does not work well: for each agent, misrepresenting own preferences is not necessarily weakly dominated by reporting own true preferences. This is because, when there are only two agents, Case 2-3 is dropped from Step 2 in the proof of Theorem 5.

As pointed out by Saijo et al. (1996), balancedness in and out of equilibrium would be a requirement that a "natural" mechanism should satisfy in pure exchange economies. So, our mechanism devised in the proof of Theorem 5 has the advantage of satisfying balancedness in and out of equilibrium. This is in contrast not only to our mechanism used to prove Theorem 3 but also to many of the mechanisms constructed in the past (e.g., Palfrey and Srivastava (1989), Jackson et al. (1994), Sjöström (1996), etc.).

Theorem 5 together with the revelation principle leads to the following corollary.

\footnotetext{
${ }^{15}$ The existence of $c, d \in C$ such that $c P_{i}^{*} d$ and $d P_{i}^{i} c$ is assured by Property 4 .

${ }^{16}$ The lexicographic tie breaking rule guarantees that $g_{j}\left(m_{i}, m_{-i}\right)=g_{j}\left(m_{i}^{\prime}, m_{-i}\right)$ even if $\operatorname{argmax} \max _{j}^{j}$ and $\arg \max _{D^{\prime}} R_{j}^{j}$ are not singletons.
} 
Corollary 4. Suppose that $n \geq 3$ and Property 4 is satisfied. Then, a social choice function is dominant strategy implementable in pure exchange economies without free disposal if and only if it satisfies strategy-proofness.

As mentioned before, Properties 1 and 2 are not necessarily satisfied in pure exchange economies without free disposal. So, even if strict value distinction holds, Corollary 3 cannot guarantee that in pure exchange economies without free disposal, every strategy-proof social choice function is implementable in dominant strategy equilibria. However, Corollary 4 tells us that even in pure exchange economies without free disposal, all strategy-proof social choice functions ${ }^{17}$ are dominant strategy implementable, provided that there are at least three agents and that strict value distinction is satisfied. Thus, it turns out that if there are three or more agents, dominant strategy implementability is not affected by whether or not free disposal is assumed.

In Example 1, we succeed in implementing a strategy-proof social choice function in dominant strategy equilibria by an augmented revelation mechanism in a two-agent pure exchange economy without free disposal. This might suggest that even in such economies, all strategy-proof social choice functions are dominant strategy implementable. However, it is an open question what is a necessary and sufficient condition for dominant strategy implementation in two-agent pure exchange economies without free disposal. This is because, as mentioned above, our mechanism devised in the proof of Theorem 5 cannot work well in such economies.

In pure exchange economies, a fixed-price social choice function (Barberà and Jackson (1995)), as well as a dictatorial one, satisfies both strategy-proofness and quasi-strong-non-bossiness; so Corollary 1 implies that these social choice functions are dominant strategy implementable via the associated direct revelation mechanisms. In contrast, an inversely dictatorial social choice function (Zhou (1991)) satisfies strategy-proofness but fails to satisfy quasi-strong-nonbossiness. It might appear that the inversely dictatorial social choice function cannot be dominant strategy implemented not only by the associated direct revelation mechanism but also by any indirect mechanism. However, it follows from Corollaries 1 and 4 that even in pure exchange economies without free disposal, every inversely dictatorial social choice function is implementable in dominant strategy equilibria. ${ }^{18}$

Like our mechanism used to prove Theorem 3, our mechanism used in the proof of Theorem 5 can boundedly implement all strategy-proof social choice

\footnotetext{
${ }^{17}$ In pure exchange economies without free disposal where there are three or more agents, Barberà and Jackson (1995) characterized the class of social choice functions that are strategy-proof, tie-free, non-bossy, and anonymous. Moreover, they pointed out that relaxing non-bossiness and/or anonymity introduces a number of substantial variations on the social choice functions characterized by them.

${ }^{18}$ Notice that in two-agent pure exchange economies without free disposal, the inversely dictatorial social choice function is equivalent to the dictatorial one.
} 
functions in undominated strategies. So, in conjunction with Corollary 1 in Jackson (1992), Theorem 5 implies Theorem 6.

Theorem 6. Suppose that $n \geq 3$ and Property 4 is satisfied. Then, a social choice function is implementable in undominated strategies by a bounded mechanism in pure exchange economies without free disposal if and only if it satisfies strategyproofness.

\section{Conclusion}

In this paper, we have considered the relationships among three notions of dominant strategy implementation especially in economic environments. Our theorems together with the revelation principle have established the following relationships in economic environments: from the point of view of implementability, (i) dominant strategy implementation via the associated direct revelation mechanism implies, but is not implied by, dominant strategy implementation by a certain indirect mechanism, and (ii) dominant strategy implementation via a certain indirect mechanism is equivalent to truthful implementation in dominant strategy equilibria. As mentioned in the introduction, when preferences involve indifference, the revelation principle might lose the powerful implication. However, the relationships tell us that in economic environments, the revelation principle has the same powerful implication as in the case when indifference is impossible.

It would be worth discussing relationships among dominant strategy implementation, Nash implementation, and bounded implementation in undominated Nash equilibria. It might seem at first glance that all of the dominant strategy implementable social choice functions are both Nash implementable and boundedly implementable in undominated Nash equilibria. However, there is no relationship among them in general. This is because, in pure exchange economies, inversely dictatorial social choice functions are dominant strategy implementable but are not boundedly implementable in undominated Nash equilibria as well as not being Nash implementable.

In this paper, we have focused mainly on economic environments. So, we leave open the question of what conditions are necessary and sufficient for dominant strategy implementation in general environments. ${ }^{19}$

\footnotetext{
${ }^{19}$ See the appendix for a partial answer to the open question.
} 


\section{Appendix}

In the appendix, by constructing a mechanism similar to the one devised to prove Theorem 3, we briefly examine a condition that is sufficient for dominant strategy implementation in general environments where there is a common worst alternative, i.e., Property 1 is satisfied. Notice that in general environments, Property 2 does not necessarily hold even if Property 1 holds. So, in general environments with a common worst alternative, there is a strategy-proof social choice function that cannot be dominant strategy implemented by any indirect mechanism even if Property 3 is satisfied, as demonstrated in Example 2.

Example 2. Consider an environment $(N, A, \mathscr{R})$ such that $N=\{1,2\}, A=\{a, b, c\}$, and $\mathscr{R}=\left\{R_{1}\right\} \times\left\{R_{2}, R_{2}^{\prime}\right\}$, where $a P_{1} c P_{1} b, a P_{2} c P_{2} b$, and $c P_{2}^{\prime} a I_{2}^{\prime} b$. A strategy-proof social choice function $f$ is given as follows.

$$
f=\begin{array}{|c|c|}
R_{2} & R_{2}^{\prime} \\
\hline a & b \\
\hline
\end{array}
$$

Note that $b$ is a common worst alternative. Note also that Property 3 holds (but Property 2 does not hold).

Now suppose that $f$ is dominant strategy implemented by an indirect mechanism $\Gamma=(M, g)$. Then, $g$ must be as follows.

\begin{tabular}{cc|ccc} 
& & \multicolumn{3}{|c}{$M_{2}$} \\
& & $D S_{2}^{\Gamma}\left(R_{2}\right)$ & $D S_{2}^{\Gamma}\left(R_{2}^{\prime}\right)$ & $\ldots$ \\
\hline \multirow{3}{*}{$M_{1}$} & $D S_{1}^{\Gamma}\left(R_{1}\right)$ & $a$ & $b$ & $\cdots$ \\
& $m_{1}^{\prime}$ & $x$ & $y$ & $\cdots$ \\
& $\vdots$ & $\vdots$ & $\vdots$ & $\ddots$
\end{tabular}

Since $D S_{2}^{\Gamma}\left(R_{2}^{\prime}\right)$ is a dominant strategy of $\Gamma$ at $R_{2}^{\prime}$, it must hold that $y P_{2}^{\prime} x$ because $b I_{2}^{\prime} a$. So, we have $y=c$, which contradicts the fact that $D S_{1}^{\Gamma}\left(R_{1}\right)$ is a dominant strategy of $\Gamma$ at $R_{1}$ because $c P_{1} b$. Thus, $f$ cannot be implemented in dominant strategy equilibria by any indirect mechanism.

Example 2 suggests that, in order to use in general environments with a common worst alternative a mechanism similar to the one used to prove Theorem 3 , we need a stronger version of Property 3 (but which is weaker than Properties 3 plus 2).

Property 5. Given $i \in N$, let $T^{i}:=\left\{a \in A \mid w R_{j} a\right.$ for all $j \in N \backslash\{i\}$ and all $\left.R_{j} \in \mathscr{R}_{j}\right\} .^{20}$ For all $i \in N$ and all $R_{i}, R_{i}^{\prime} \in \mathscr{R}_{i}$, if $R_{i} \neq R_{i}^{\prime}$, then there exist $a, b \in T^{i} \subseteq A$ such that $a P_{i} b, b P_{i}^{\prime} a, a P_{i} w$, and $b P_{i}^{\prime} w$.

Theorem 7. Suppose that Properties 1 and 5 are satisfied. Then, if a social choice function $f$ satisfies strategy-proofness, then it is dominant strategy implementable in general environments.

\footnotetext{
${ }^{20}$ Note that $T^{i} \neq \varnothing$ for every $i \in N$.
} 
Proof. Fix an arbitrary $w \in A$ that satisfies Property 1 . We construct an augmented revelation mechanism $\Gamma=(M, g)$ which is similar to the one constructed in the proof of Theorem 3. Agent $i$ 's message space is

$$
M_{i}:=\mathscr{R}_{i} \cup\left(T^{i+1} \times T^{i+1}\right),
$$

where $T^{i+1}=\left\{a \in A \mid w R_{j} a\right.$ for all $j \in N \backslash\{i+1\}$ and all $\left.R_{j} \in \mathscr{R}_{j}\right\}$. A typical message for agent $i \in N$ is denoted by $m_{i}=R_{i}^{i}$ or $\left(a^{i}, b^{i}\right)$. Let $g$ be defined as follows. Rule 1: If $m_{i}=R_{i}^{i} \in \mathscr{R}_{i}$ for all $i \in N$, then $g(m):=f\left(R_{1}^{1}, R_{2}^{2}, \ldots, R_{n}^{n}\right)$. Note that Property 1 assures that $g(m) R_{i}^{i} w$ for all $i \in N$.

Rule 2: If $m_{i}=R_{i}^{i} \in \mathscr{R}_{i}$ for some $i \in N$ and $m_{j}=\left(a^{j}, b^{j}\right) \in\left(T^{j+1} \times T^{j+1}\right)$ for all $j \neq i$, then $g(m):=\arg \max _{\left\{a^{i-1}, b^{i-1}, w\right\}} R_{i}^{i}$. Ties are broken in some arbitrary manner unless $\arg \max _{\left\{a^{i-1}, b^{i-1}, w\right\}} R_{i}^{i}$ is a singleton. Notice that $g(m) R_{i}^{i} w$ and $w R_{j}^{\prime} g(m)$ for all $j \neq i$ and all $R_{j}^{\prime} \in \mathscr{R}_{j}$ by construction.

Rule 3: In all other cases, $g(m):=w$.

A reasoning similar to that in the proof of Theorem 3 establishes that $g\left(D S E^{\Gamma}(R)\right)=$ $f(R)$ for all $R \in \mathscr{R}$.

When coupled with the revelation principle, Theorem 7 leads to the following corollary.

Corollary 5. Suppose that Properties 1 and 5 are satisfied. Then, a social choice function is dominant strategy implementable in general environments if and only if it satisfies strategy-proofness.

In the appendix, we concentrate on general environments where there is a common worst alternative, and explore a sufficient condition for dominant strategy implementation by using a mechanism similar to the one constructed to prove Theorem 3. So, Corollary 5 is a partial answer to the open question mentioned in the conclusion. It leaves open the question of when Property 5 is relaxed, whether or not it is possible for a mechanism that may be fundamentally different from the one used in the proof of Theorem 7 to dominant strategy implement every strategy-proof social choice function in general environments with a common worst alternative. It also leaves open the question of what conditions are necessary and sufficient for dominant strategy implementation in general environments without a common worst alternative. 


\section{References}

BARBERÀ, S. AND M. O. JACKSON (1995): "Strategy-Proof Exchange," Econometrica, 63, 51-87.

Bergemann, D. AND S. Morris (2005): "Robust Implementation: The Role of Large Type Spaces,” mimeo., Yale University.

DAsGupta, P., P. J. HAMmOND, AND E. MASKIN (1979): "The Implementation of Social Choice Rules: Some General Results on Incentive Compatibility," Review of Economic Studies, 46, 185-216.

JACKSON, M. O. (1992): "Implementation in Undominated Strategies: A Look at Bounded Mechanisms,” Review of Economic Studies, 59, 757-775.

(2001): "A Crash Course in Implementation Theory," Social Choice and Welfare, 18, 655-708.

Jackson, M. O., T. R. Palfrey, and S. SRivastava (1994): "Undominated Nash Implementation in Bounded Mechanisms," Games and Economic Behavior, 6, 474-501.

LAFFONT, J.-J. AND E. MASKIN (1982): "Nash and Dominant Strategy Implementation in Economic Environments," Journal of Mathematical Economics, 10, $17-47$.

Mas-Colell, A., M. D. Whinston, And J. R. Green (1995): Microeconomic Theory, Oxford University Press.

Mookherjee, D. And S. Reichelstein (1990): "Implementation via Augmented Revelation Mechanisms,” Review of Economic Studies, 57, 453-475.

PALFREY, T. R. AND S. SRIVASTAVA (1989): "Implementation with Incomplete Information in Exchange Economies," Econometrica, 57, 115-134.

Postlewaite, A. And D. Schmeidler (1986): "Implementation in Differential Information Economies," Journal of Economic Theory, 39, 14-33.

Repullo, R. (1986): "On the Revelation Principle under Complete and Incomplete Information," in Economic Organization as Games, ed. by K. Binmore and P. Dasgupta, Basil Blackwell, 179-195.

RitZ, Z. (1983): "Restricted Domains, Arrow-Social Welfare Functions and Noncorruptible and Non-manipulable Social Choice Correspondences: The Case of Private Alternatives," Mathematical Social Sciences, 4, 155-179.

SAIJO, T. (2005): "Spiteful Behavior in Voluntary Contribution Mechanism Experiments," in Handbook of Experimental Economics Results, ed. by C. R. Plott and V. L. Smith, North-Holland. 
SAijo, T., T. SjÖströM, AND T. YAmato (2005): "Secure Implementation," mimeo., Osaka University.

SAijO, T., Y. TATAMitAni, AND T. YAMATO (1996): “Toward Natural Implementation," International Economic Review, 37, 949-980.

SJÖSTRÖM, T. (1994): "Implementation in Undominated Nash Equilibria without Integer Games," Games and Economic Behavior, 6, 502-511.

(1996): "Implementation by Demand Mechanisms," Economic Design, 1, 343-354.

ZHOU, L. (1991): "Inefficiency of Strategy-Proof Allocation Mechanisms in Pure Exchange Economies," Social Choice and Welfare, 8, 247-254. 